\title{
CORRECTION
}

\section{Correction to: PIK3R5 genetic predictors of hypertension induced by VEGF-pathway inhibitors}

Julia C. F. Quintanilha (D), Alessandro Racioppi, Jin Wang, Amy S. Etheridge, Stefanie Denning, Carol E. Peña, Andrew D. Skol,

Daniel J. Crona (D), Danyu Lin and Federico Innocenti iD

(c) The Author(s), under exclusive licence to Springer Nature Limited 2021

The Pharmacogenomics Journal (2022) 22:145; https://doi.org/10.1038/s41397-021-00264-2

Correction to: The Pharmacogenomics Journal https://doi.org/ 10.1038/s41397-021-00261-5, 13 November 2021

In the original version of the article, the Funding Information was revised. The original article has been corrected.

\section{FUNDING}

This work was supported by the National Cancer Institute of the National Institutes of Health under Award Numbers U10CA180821 and U10CA180882 (to the Alliance for Clinical Trials in Oncology), U24CA196171, R21CA139280-01, and K07CA140390-01. JCFQ was supported by the São Paulo Research Foundation (FAPESP 2018/04491-2). 